AIAA 2003-7062

\title{
SIMULATION OF SHARP LEADING EDGE AEROTHERMODYNAMICS
}

\author{
Iain D. Boyd ${ }^{*}$ and Jose F. Padilla ${ }^{\dagger}$ \\ University of Michigan, Ann Arbor, Michigan, 48109
}

\begin{abstract}
Future hypersonic vehicles are likely to use sharp leading edges on wings and engine inlets to reduce drag. The radius of curvature associated with such structures may be one centimeter or less. The very small size leads to high heating rates that can be accommodated using advanced materials. An additional aspect of the small size concerns the basic gas dynamics. At such small scales, the continuum approach invoked in formulating the Navier-Stokes equations may be invalid. In this study, the flow around various sharp leading edge shapes is computed for a high-altitude point on a typical re-entry trajectory. The particle-based direct simulation Monte Carlo method (DSMC) is employed. Effects are investigated of variation in free stream velocity and leading edge shape in terms of flow field and surface properties. The aerothermodynamic performance of the sharp leading edges is assessed using the shock standoff distance, the total drag, and the heat transfer rate. The data generated in the present study form the initial part of a more complete investigation involving the optimization of leading edges over a hypersonic re-entry trajectory.
\end{abstract}

\section{Introduction}

Air-breathing, hypersonic vehicles are being considered for use as reusable launch vehicles (RLV's) and other trans-atmospheric aircraft. Among the primary technology issues concerning the aerodynamics of such vehicles is the need to minimize overall vehicle drag. One promising approach involves the use of sharp leading edges for lifting surfaces and engine inlets defined using power-law shapes [1]. Prior theoretical and analytical studies have focused attention on different flight regimes. For example, O'Brien and Lewis [2] considered very low altitude, inviscid conditions by solving the Euler equations, while Santos and Lewis [3,4] applied the direct simulation Monte Carlo method (DSMC) [5] to study high altitude, rarefied conditions. The present work has as its goal a more comprehensive computational analysis, using various numerical methods, of the aerodynamic performance of sharp leading edges across the full range of conditions experienced in a representative flight trajectory. The assessment will involve comparisons of heat transfer, drag force, and shock standoff distance, for a variety of on- and offdesign points.

To first order, the trajectory of an air-breathing hypersonic reusable launch vehicle is designed to provide pressures inside the propulsion system to be a sizeable fraction of atmospheric pressure in order to achieve efficient combustion. For the present numerical studies, we use a typical air-breathing vehicle trajectory provided by Bertin [6]. For this trajectory, Fig. 1a shows the velocity and dynamic pressure as a function of altitude. The latter quantity provides a good estimate of the pressure that can be generated inside the propulsion system. For this trajectory, it is clear that efficient combustion is not available until an altitude of about $45 \mathrm{~km}$. In the descent from orbit, the RLV would enter on an equilibrium glide trajectory similar to the Space Shuttle and the engine, a scramjet in this case, would be ignited at about $45 \mathrm{~km}$. In Fig. 1b, profiles of stagnation point heat flux and Knudsen number are shown for the same trajectory. The heat flux in $\mathrm{MW} / \mathrm{m}^{2}$ is determined using the following correlation of Detra et al. [7]:

$$
\dot{q}=\frac{110.3}{\sqrt{R_{N}}} \sqrt{\frac{\rho}{\rho_{S L}}}\left(\frac{U}{U_{C O}}\right)^{3.15}
$$

where $R_{N}$ is the nose radius, taken to be $5 \mathrm{~mm}$ representative of the sharp leading edges of interest in this study, $\rho_{\mathrm{SL}}$ is sea-level mass density, and $U_{\mathrm{CO}}$ is the orbital velocity (taken to be $7.95 \mathrm{~km} / \mathrm{s}$ ). The Knudsen number is computed using a constant, hard-sphere collision cross section, and a characteristic length of $5 \mathrm{~mm}$. Note that, as the vehicle descends through the atmosphere, the Knudsen number decreases from the rarefied flow regime $\left(10^{-1}\right)$ through the transition regime into the continuum flow regime $\left(10^{-4}\right)$. While the region of maximum heat flux (around $40 \mathrm{~km}$ ) has a Knudsen number in the nearcontinuum regime (about $5 \times 10^{-3}$ ), the local radius of curvature on a sharp leading edge may be smaller than the gross dimension of $5 \mathrm{~mm}$ thus increasing the local Knudsen number. Hence, the overall assessment of sharp leading edges for such vehicles requires both continuum and rarefied flow analyses. In our work, the continuum regions will be analyzed using the Navier-Stokes equations and the rarefied regions will be computed using the DSMC technique.

In this paper, we present our initial results from this long-term study. We elect to begin our analysis at a high altitude condition due to our familiarity with this regime, and due to the availability of previous DSMC results of Santos and Lewis $[3,4]$. In the next section, we describe the DSMC technique and the particular code and models used in the present study. Then, we present the various flow conditions and sharp leading edge geometries that are investigated. Results

\footnotetext{
* Professor. Department of Aerospace Engineering. Associate Fellow AIAA.

${ }^{\dagger}$ Graduate Student. Department of Aerospace Engineering.
} 
from the simulations are then presented. Finally, we end with a summary and concluding remarks.

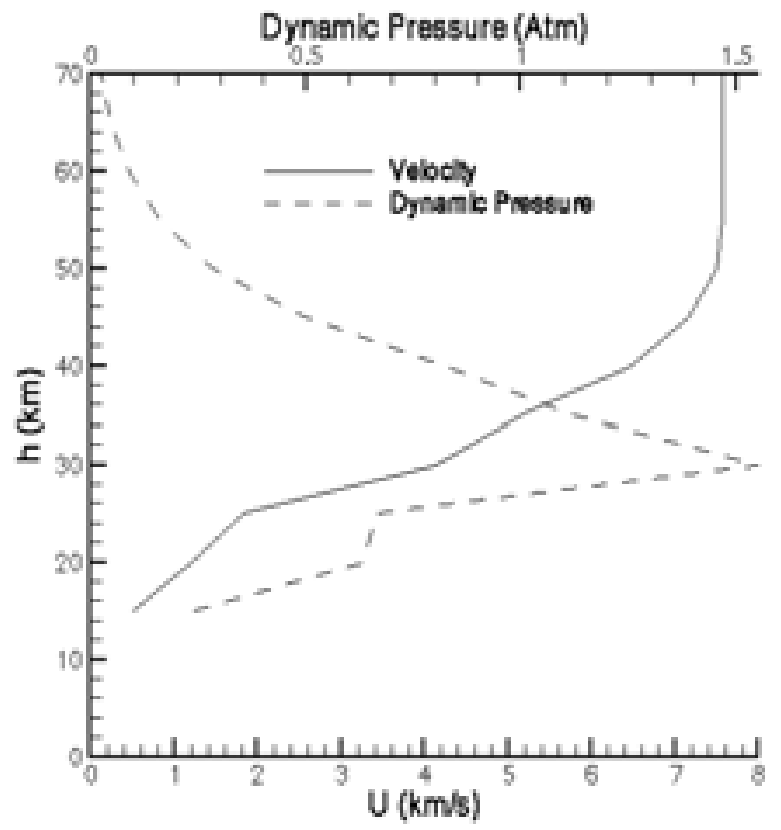

Fig. 1a. Profiles of velocity and dynamic pressure on a representative, air-breathing RLV trajectory.

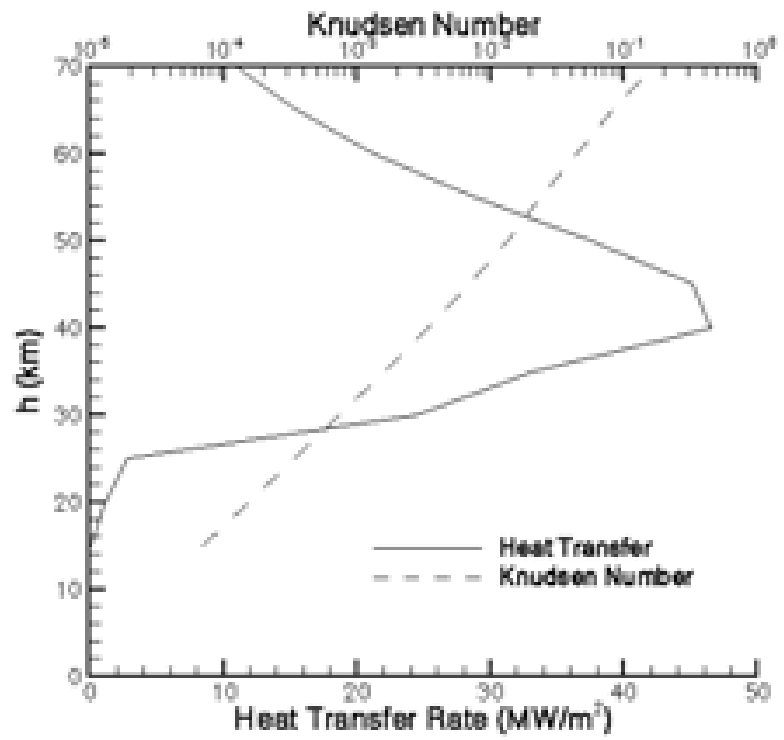

Fig. 1b. Profiles of stagnation point heating and Knudsen number on a representative, air-breathing RLV trajectory.

\section{Numerical Method}

As discussed above, the DSMC technique is used in this study to compute rarefied hypersonic flows around sharp leading edges. The DSMC code employed in the present study is called MONACO and is a general, object-oriented, cellbased, parallelized implementation of the DSMC method first developed by Dietrich and Boyd [8]. Parallelization is performed using domain decomposition. In the original study [8], a 400-processor computation on the first IBM SP-2 supercomputer was performed using MONACO using 100 million particles. Since that time, MONACO has been further developed and applied to a range of rarefied gas problems including hypersonic viscous interactions [9], threedimensional spacecraft plume studies [10], spacecraft glow modeling [11], thin film deposition [12], and meteoroid trail analysis [13].

In terms of physical models, MONACO employs the Variable Soft Sphere (VSS) collision model [14], the variable rotational energy exchange probability model of Boyd [15], and the variable vibrational energy exchange probability model of Vijayakumar et al. [16]. Chemical reactions are included in the present analysis and are simulated using the Total Collision Energy (TCE) model of Bird [5]. A 5-species air chemistry reaction mechanism is implemented. Simulation of the interactions of the particles with the walls employs accommodation coefficients to specify the fraction of diffuse and specular reflections.

MONACO offers a lot of control over the numerical parameters employed in a simulation. Both unstructured triangular, or structured rectangular cells may be employed in two-dimensional simulations. Cell weighting factors and timesteps may be set uniquely for each cell in the grid. A sub-cell system is implemented for selection of collision pairs where the number of sub-cells is scaled by the local mean free path.

\section{Results}

MONACO is applied to study four different cases (listed in Table 1) at an altitude of $70 \mathrm{~km}$ where the free stream atmospheric conditions are: $\rho_{\infty}=8.3 \times 10^{-5} \mathrm{~kg} / \mathrm{m}^{3}$, and $\mathrm{T}_{\infty}=220 \mathrm{~K}$. Two different free stream velocities are considered: (1) 3.5 $\mathrm{km} / \mathrm{s}$, corresponding to the conditions studied by Santos and Lewis $[3,4]$; and (2) $7.5 \mathrm{~km} / \mathrm{s}$, corresponding to the point at 70 km shown on the trajectory of Fig. 1. Three different leading edge shapes are considered, the first of which is the nominal case of a cylinder. The two other cases are derived from the following simple power-law form as used in Refs. 3, 4:

$$
\mathrm{y}=\mathrm{a} \mathrm{x}^{\mathrm{n}}
$$

where $\mathrm{x}$ and $\mathrm{y}$ are the two-dimensional coordinates, and we consider the cases of $n=0.5$ and $n=0.7$ with a maximum height of $1 \mathrm{~cm}$. The three different leading edge shapes are compared in Fig. 2. Notice that the power law shapes increase the degree of sharpness of the leading edge in comparison to the nominal cylinder. The wall temperature is fixed at $880 \mathrm{~K}$ and fully diffuse reflection is assumed again for compatibility with Refs. 3 and 4.

Table 1. Cases considered.

\begin{tabular}{ccc}
\hline Case & Shape & $\mathrm{U}_{\infty}(\mathrm{km} / \mathrm{s})$ \\
\hline 1 & Cylinder & 3.5 \\
2 & Cylinder & 7.5 \\
3 & $\mathrm{n}=0.5$ & 7.5 \\
4 & $\mathrm{n}=0.7$ & 7.5 \\
\hline
\end{tabular}


The solution procedure adopted is as follows: first, a coarse simulation is performed with a relatively low number of particles; then these results are used to generate a new mesh that is adapted to the spatial distribution of local mean free path. For illustration, the final adapted meshes obtained in this way at $7.5 \mathrm{~km} / \mathrm{s}$ for the cylinder and for the $\mathrm{n}=0.7$ powerlaw shape are shown in Figs. 3a and 3b, respectively. In these computational domains, $\mathrm{y}=0$ is a plane of symmetry, the left hand edge of the mesh is an inflow boundary, the $x=x_{\max }$ plane is an outflow boundary, and the wall is fully diffuse. As expected, the mesh is clustered towards the surface due to gas compression leading to a reduction in the mean free path. Note that the level of refinement required for the blunter cylinder configuration is significantly greater than that for the more oblique power law shape. For the various cases computed, the total number of cells is of the order of 10,000 and the total number of particles is 300,000 . The time step in all simulations is of the order of $10^{-9} \mathrm{sec}$ that is a small fraction of the lowest mean free time. The simulations are typically run for several hundred thousand iterations to ensure full convergence. Contours of temperature ratio for these same two cases are shown in Figs. $4 a$ and $4 b$ to illustrate the very different flow fields produced by these two different leading edge shapes.

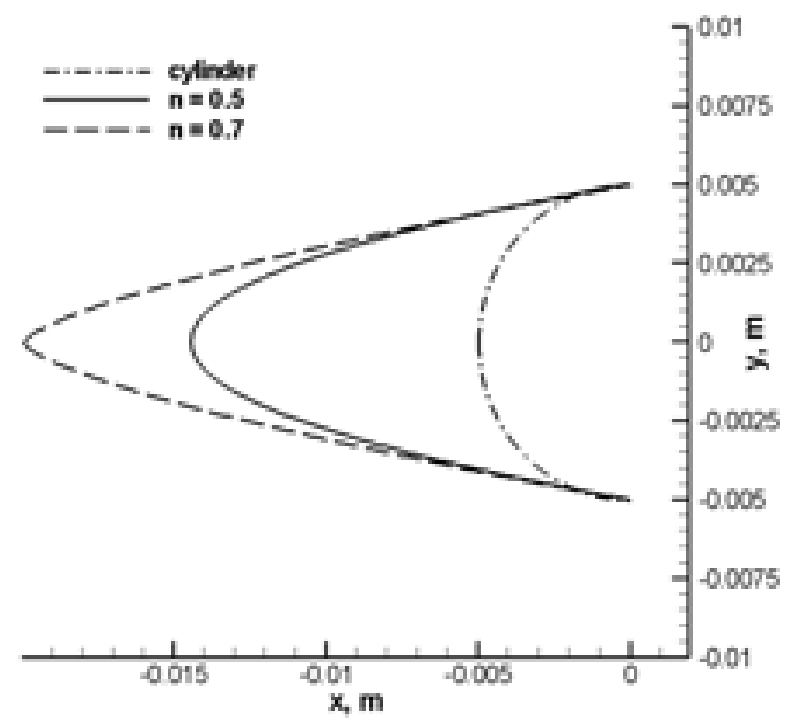

Fig. 2. Sharp leading edge shapes considered.

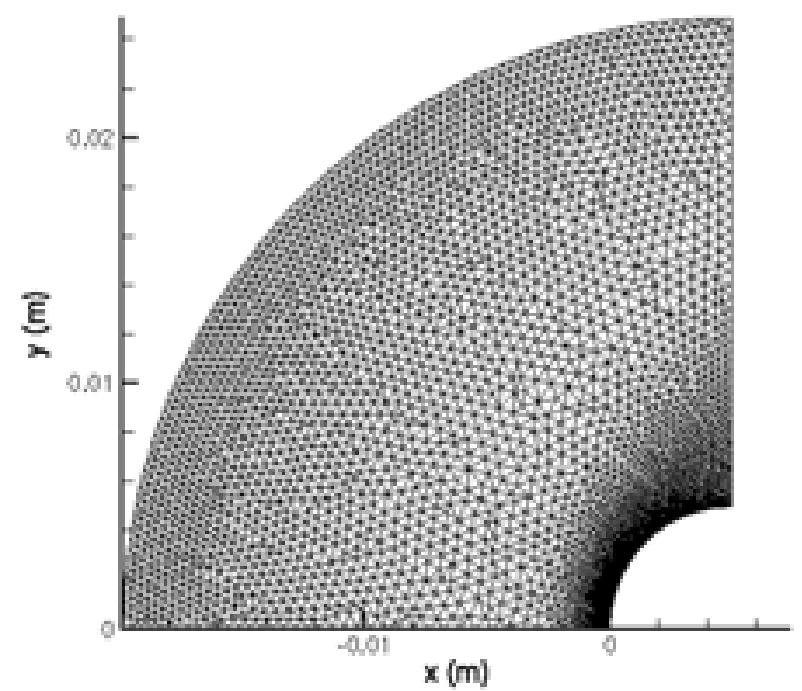

Fig. 3a. Adapted computational mesh for flow around cylinder at $\mathrm{U}_{\infty}=7.5 \mathrm{~km} / \mathrm{s}$.

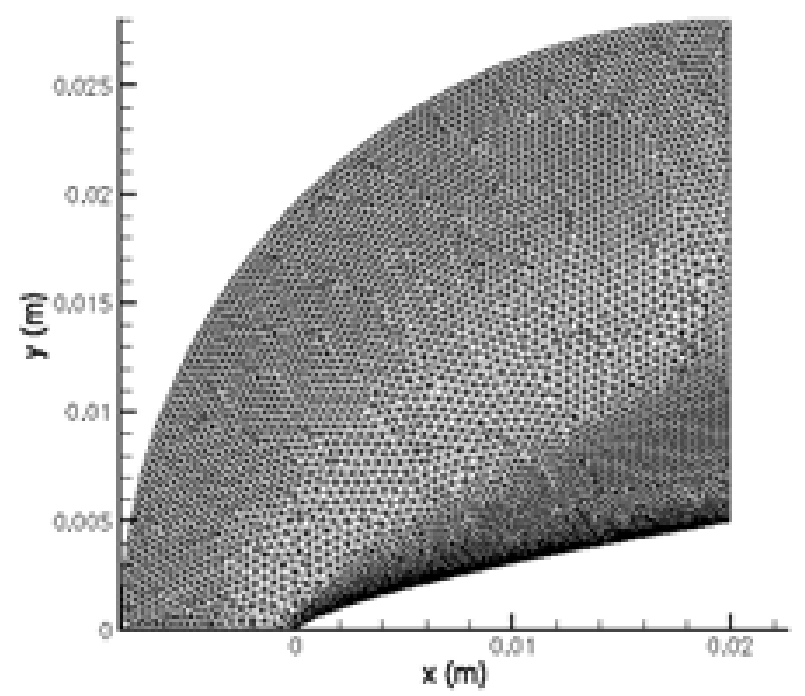

Fig. 3b. Adapted computational mesh for flow around $n=0.7$ power-law shape at $U_{\infty}=7.5 \mathrm{~km} / \mathrm{s}$. 


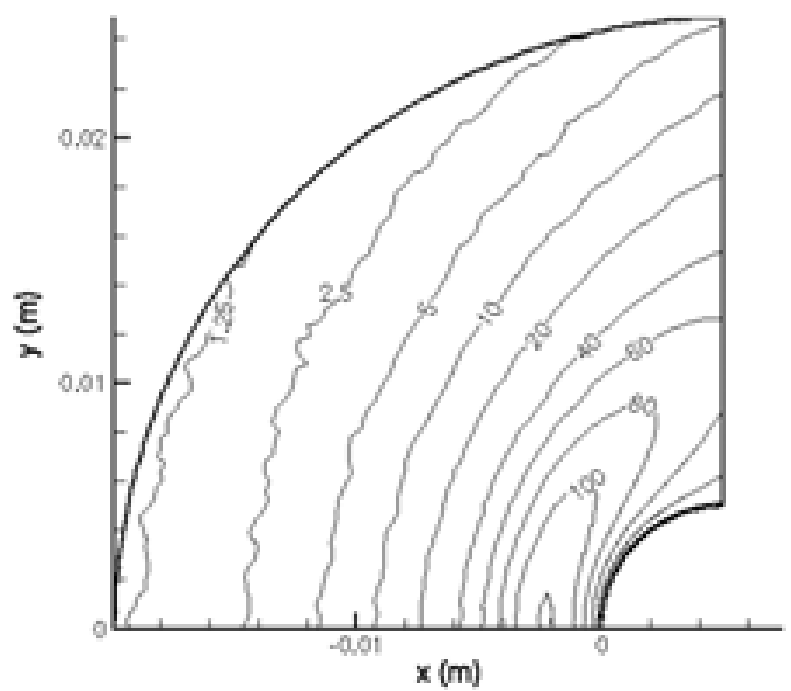

Fig. 4a. Contours of temperature ratio $\left(\mathrm{T} / \mathrm{T}_{\infty}\right)$ for flow around cylinder at $\mathrm{U}_{\infty}=7.5 \mathrm{~km} / \mathrm{s}$.

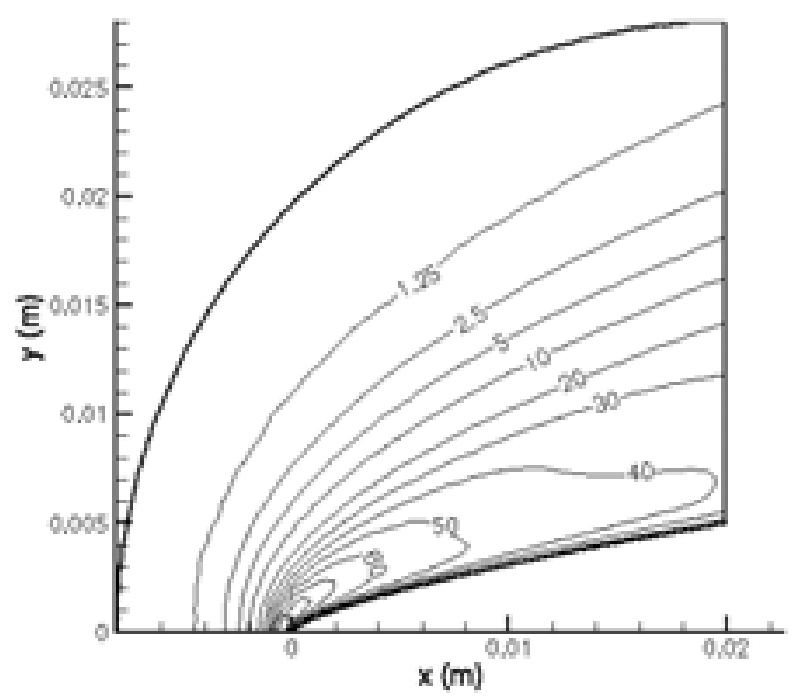

Fig. 4b. Contours of temperature ratio $\left(\mathrm{T} / \mathrm{T}_{\infty}\right)$ for flow around $\mathrm{n}=0.7$ power-law shape at $\mathrm{U}_{\infty}=7.5 \mathrm{~km} / \mathrm{s}$.

\subsection{Effect of Velocity on Cylindrical Leading Edge}

We first consider the effect of free stream velocity on the flow around the cylindrical leading edge. Profiles obtained at free stream velocities of 3.5 and $7.5 \mathrm{~km} / \mathrm{s}$ are compared along the stagnation streamline for several different flow properties in Figs. 5a through 5e. The profiles of density ratio for the two cases shown in Fig. 5a are nearly identical. A working definition for shock standoff distance in nonequilibrium hypersonic flows is the point at which the density ratio is equal to six (this is the asymptotic value from perfect diatomic gas normal shock theory at infinite Mach number). Using this definition, the profiles of Fig. 5a indicate that the shock standoff distance is unchanged by the dramatic increase in free stream velocity. The profiles of velocity ratio in Fig. $5 \mathrm{~b}$ are also almost identical for the two cases. The pressure ratio profiles in Fig. 5c, as expected, indicate a significantly higher pressure for the higher velocity case. Figures $5 \mathrm{~d}$ and $5 \mathrm{e}$ show the temperature ratio profiles for the translational, rotational, and vibrational energy modes for Cases 1 and 2, respectively. The profiles for Case 1 shown in Fig. 5d indicate significant thermal nonequilibrium between the energy modes. The extent of nonequilibrium is even greater for Case 2 as is the distance from the wall at which the translational temperature first starts to increase. It should be noted that most of the current DSMC results at $3.5 \mathrm{~km} / \mathrm{s}$ are in excellent agreement with the profiles reported for the same conditions in Refs. 3 and 4. The one exception is the temperature profiles where use of the more detailed rotational and vibrational relaxation models in MONACO [15,16] lead to lower rotational and vibrational temperatures.

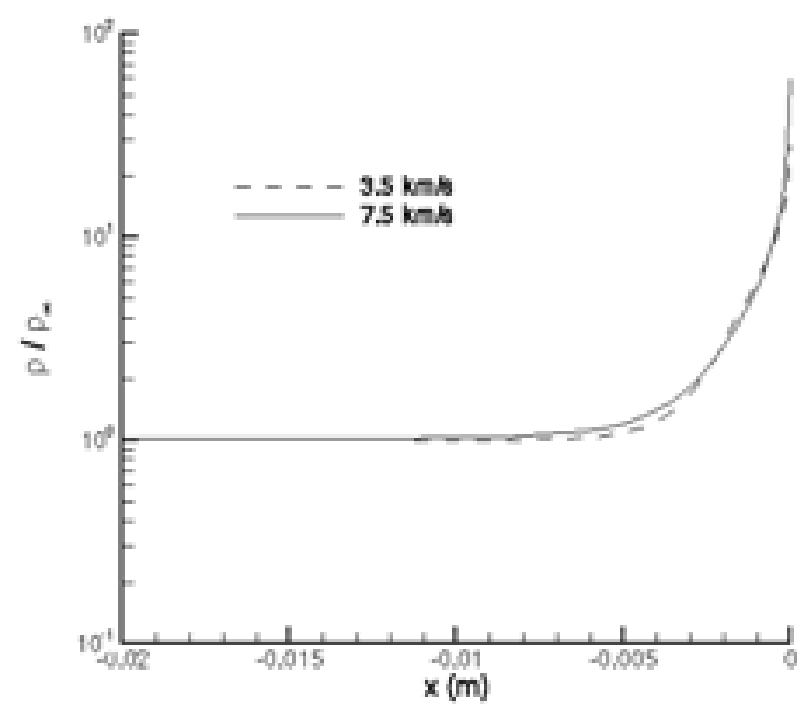

Fig. 5a. Density profiles along the cylinder stagnation streamline at 2 different velocities. 


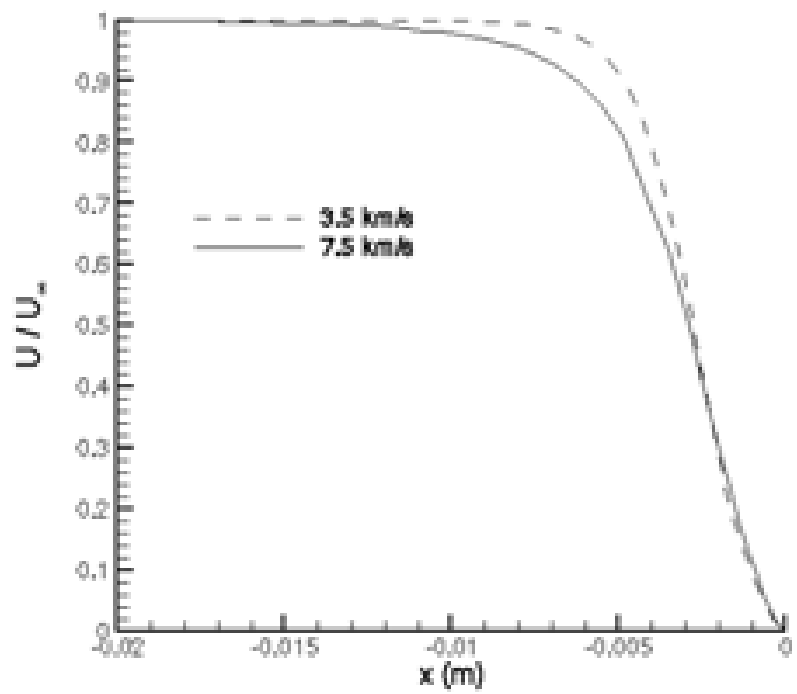

Fig. 5b. Velocity profiles along the cylinder stagnation streamline at 2 different velocities.

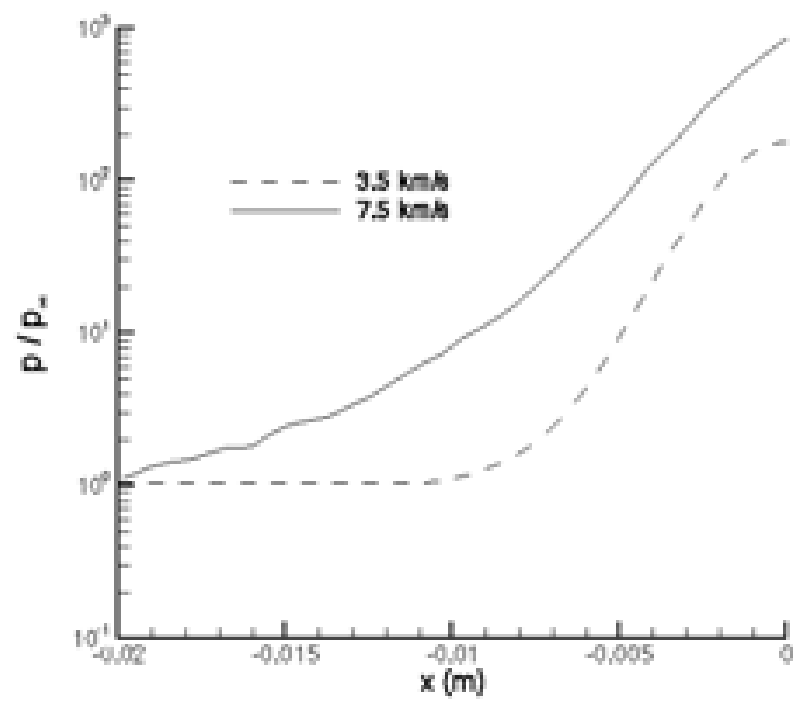

Fig. 5c. Pressure profiles along the cylinder stagnation streamline at 2 different velocities.

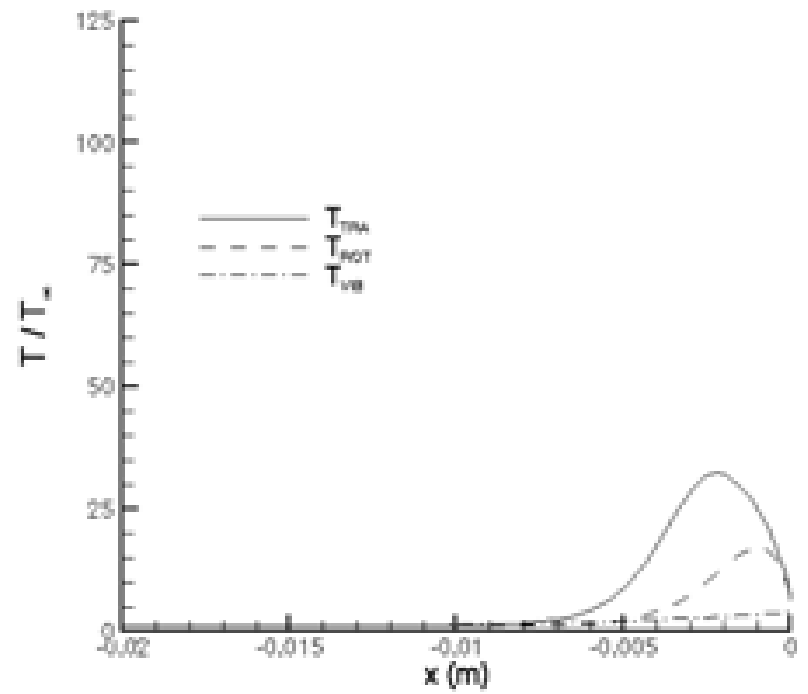

Fig. 5d. Temperature profiles along the stagnation streamline at $\mathrm{U}_{\infty}=3.5 \mathrm{~km} / \mathrm{s}$ for the cylinder.

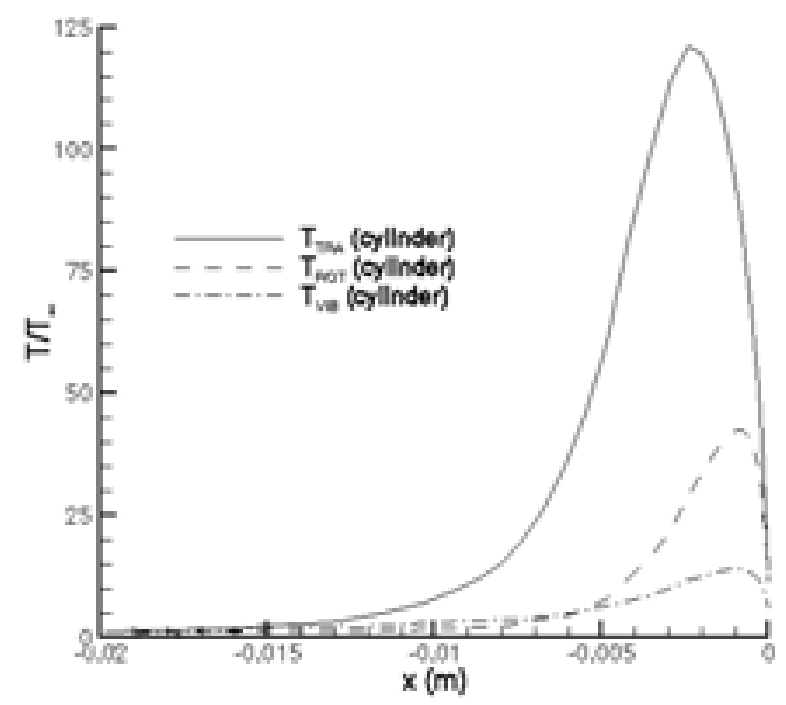

Fig. 5e. Temperature profiles along the stagnation streamline at $\mathrm{U}_{\infty}=7.5 \mathrm{~km} / \mathrm{s}$ for the cylinder.

Profiles of the surface properties for the two conditions are compared in Figs. 6a through $6 \mathrm{c}$. The following standard definitions are employed for the pressure, skin friction, and heat transfer coefficients:

$$
C_{p} \equiv \frac{p_{w}-p_{\infty}}{\frac{1}{2} \rho_{\infty} U_{\infty}^{2}}
$$




$$
\begin{aligned}
C_{f} & \equiv \frac{\tau_{w}}{\frac{1}{2} \rho_{\infty} U_{\infty}^{2}} \\
C_{h} \equiv & \frac{\dot{q}}{\frac{1}{2} \rho_{\infty} U_{\infty}^{3}}
\end{aligned}
$$

where $p_{w}$ is the wall pressure, $\tau_{w}$ is the wall shear stress and $\dot{q}$ is the heat transfer rate. The dependent variable is the $\mathrm{x}$ coordinate along the surface divided by the body radius, $\mathrm{R}=0.005 \mathrm{~m}$. Once again it should be noted that the present DSMC results at $3.5 \mathrm{~km} / \mathrm{s}$ are in excellent agreement with the previous profiles at the same conditions reported in Refs. 3 and 4. The two profiles of pressure coefficient in Fig. 6a are almost identical and closely follow Newtonian theory. Both the skin friction and the heat transfer coefficient are smaller for the lower velocity case. The stagnation point heat transfer correlation of Detra et al. [7], Eq. (1), predicts values of $C_{h}$ for these conditions of 0.42 and 0.62 , at velocities of 3.5 and 7.5 $\mathrm{km} / \mathrm{s}$, respectively. While these values exceed those from the simulations by $20-40 \%$, Eq. (1) does predict that a higher free stream velocity should produce a higher heat transfer coefficient in qualitative agreement with the simulation results.

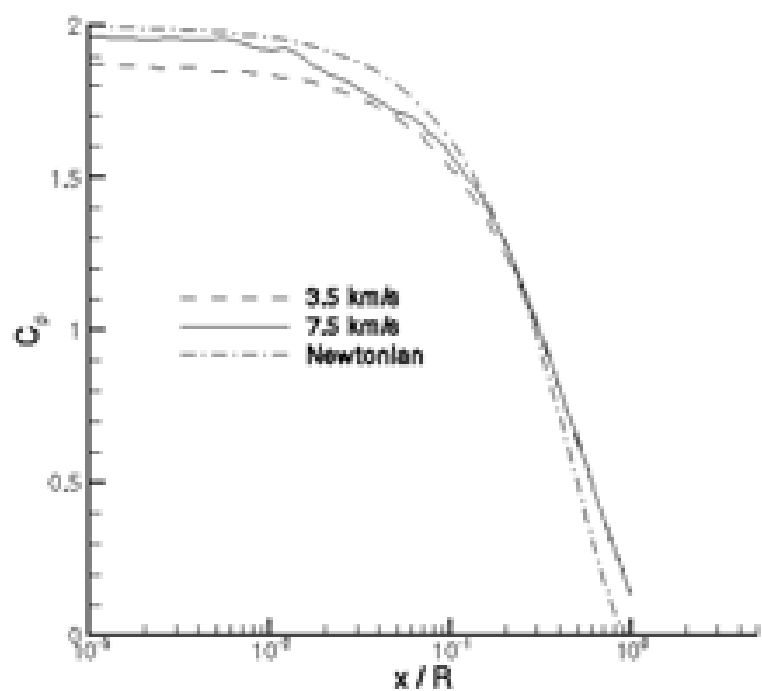

Fig. 6a. Surface profiles of pressure coefficient around the cylinder.

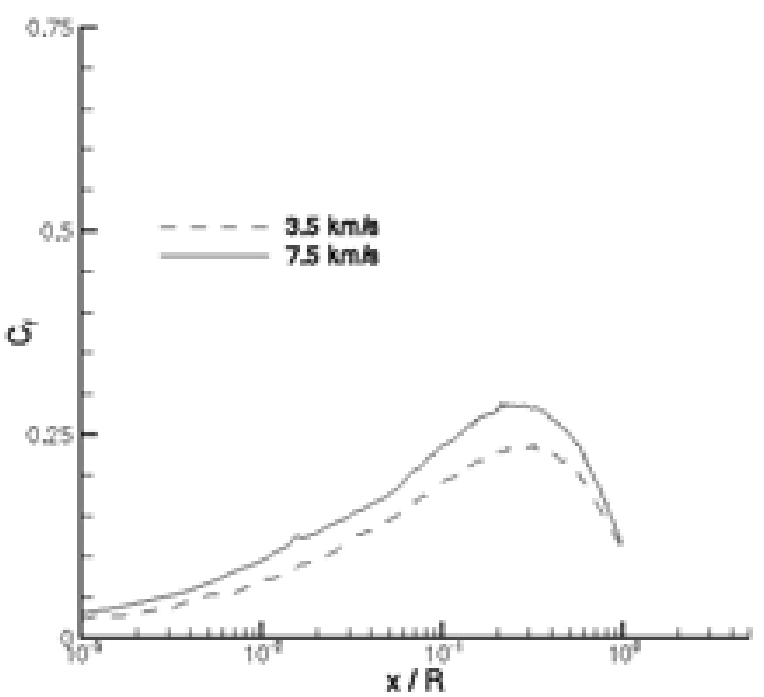

Fig. 6b. Surface profiles of skin friction coefficient around the cylinder.

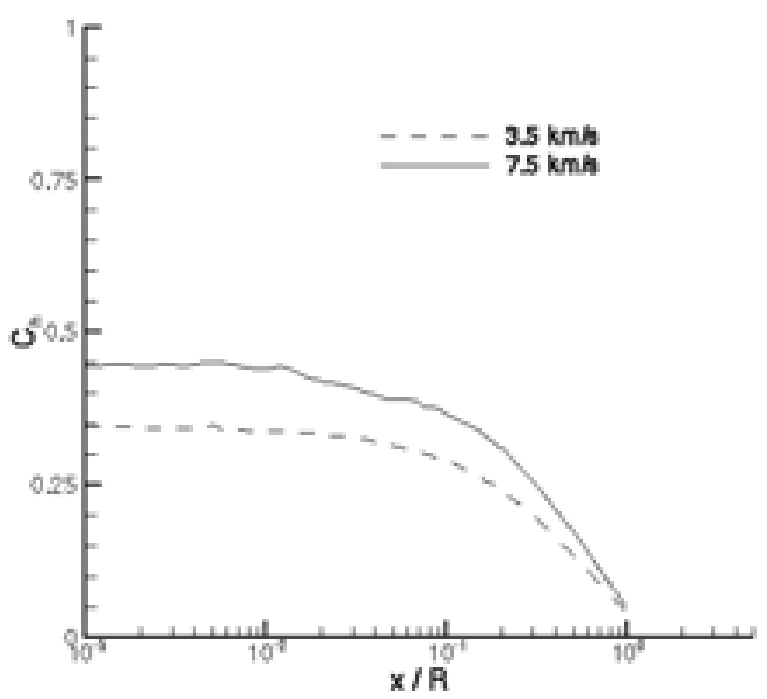

Fig. 6c. Surface profiles of heat transfer coefficient around the cylinder.

\subsection{Effect of Leading Edge Shape}

We next consider the effect of variation in the leading edge shape for the free stream velocity of $7.5 \mathrm{~km} / \mathrm{s}$. Figures $7 \mathrm{a}$ through $7 \mathrm{~g}$ compare stagnation streamline profiles for the cylinder, and $\mathrm{n}=0.5$ and $\mathrm{n}=0.7$ power-law shapes, respectively. The density ratio profiles shown in Fig. 7a reveal significant reductions in the stagnation point density as the leading edge is sharpened. Again using the definition of $\rho / \rho_{\infty}=6$ as the shock standoff distance, it is clear that the shock is barely detached for the $\mathrm{n}=0.7$ shape. Minimization of the shock standoff distance is desirable to avoid flow spillage around the upper surface of the leading edge that would decrease lift on a 
wing and decrease engine inlet flow rate on a cowl lip, both of which reduce the vehicle efficiency. The velocity and pressure profiles, shown in Figs. $7 \mathrm{~b}$ and $7 \mathrm{c}$, further illustrate the differences in the compression processes for the three leading edge shapes. The three sets of temperature profiles shown in Figs. $7 \mathrm{~d}$ through $7 \mathrm{f}$ illustrate the strong thermal nonequilibrium effects and the significantly reduced compression for the $n=0.7$ case. The high temperature ratios generated in the cylinder flow are sufficient to cause a significant amount of molecular oxygen dissociation as illustrated in Fig. $7 \mathrm{~g}$ that shows the stagnation streamline mole fraction profiles for this case. The $n=0.7$ power-law shape generated significantly less chemistry.

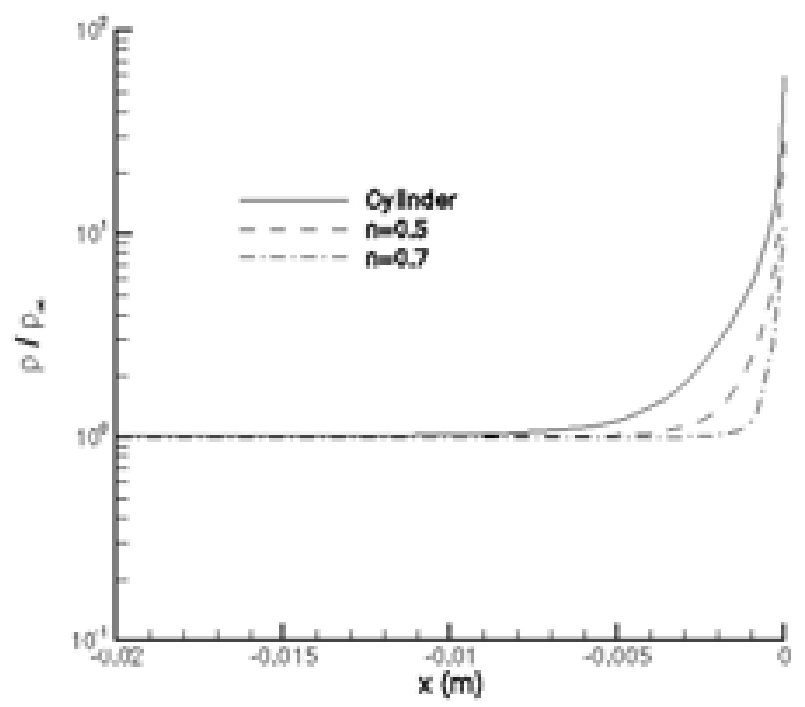

Fig. 7a. Density profiles along the stagnation streamline at $\mathrm{U}_{\infty}=7.5 \mathrm{~km} / \mathrm{s}$.

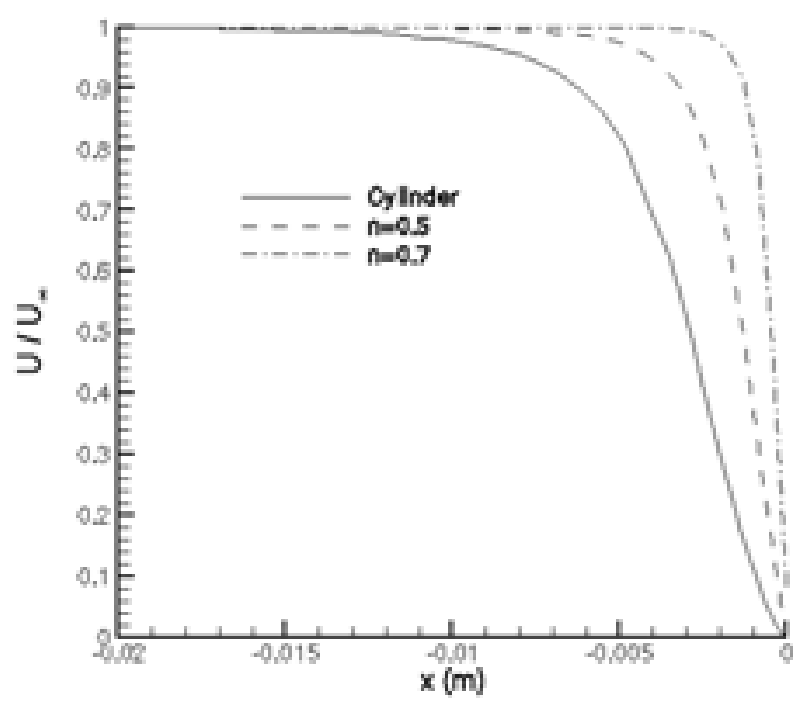

Fig. 7b. Velocity profiles along the stagnation streamline at $\mathrm{U}_{\infty}=7.5 \mathrm{~km} / \mathrm{s}$.

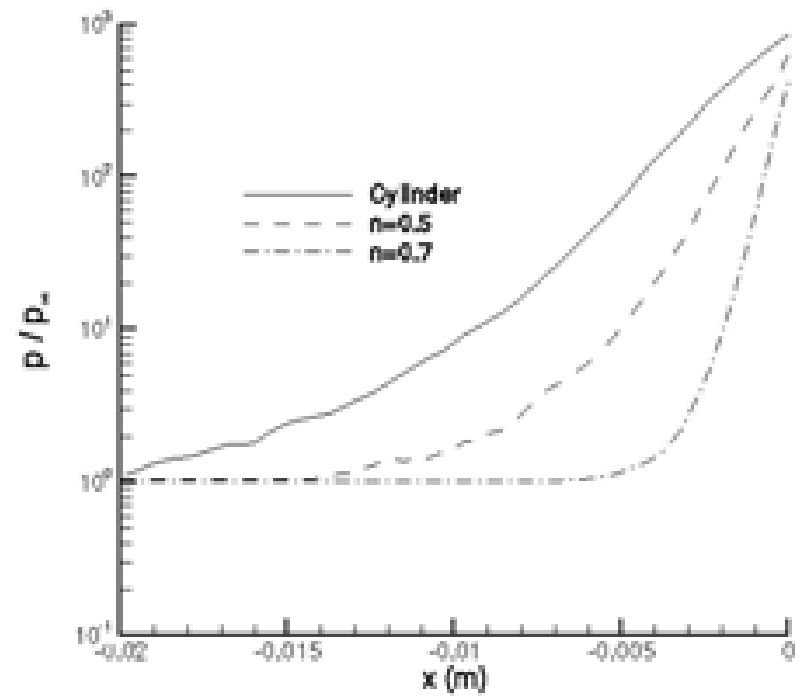

Fig. 7c. Pressure profiles along the stagnation streamline at $\mathrm{U}_{\infty}=7.5 \mathrm{~km} / \mathrm{s}$.

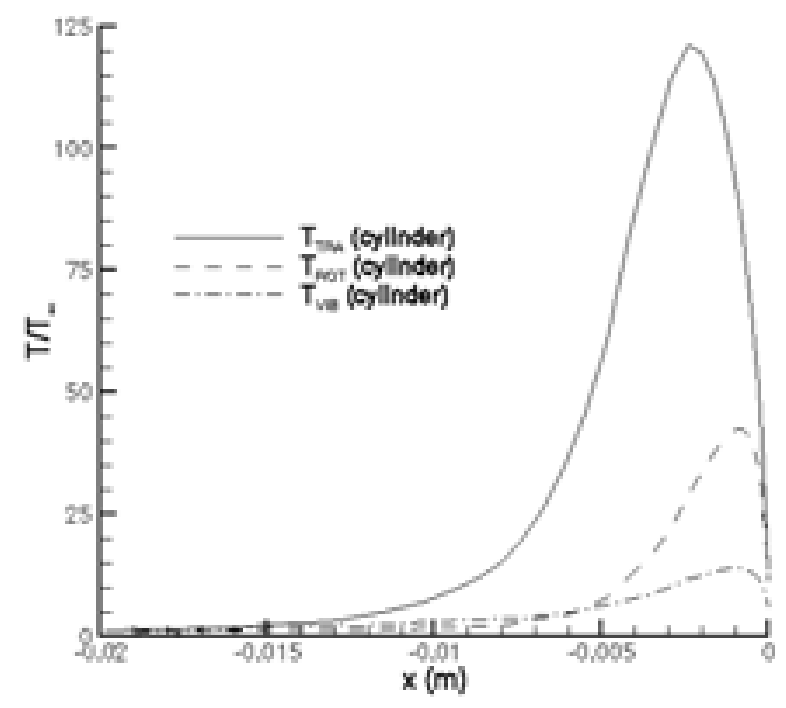

Fig. 7d. Temperature profiles along the stagnation streamline at $\mathrm{U}_{\infty}=7.5 \mathrm{~km} / \mathrm{s}$ for the cylinder. 


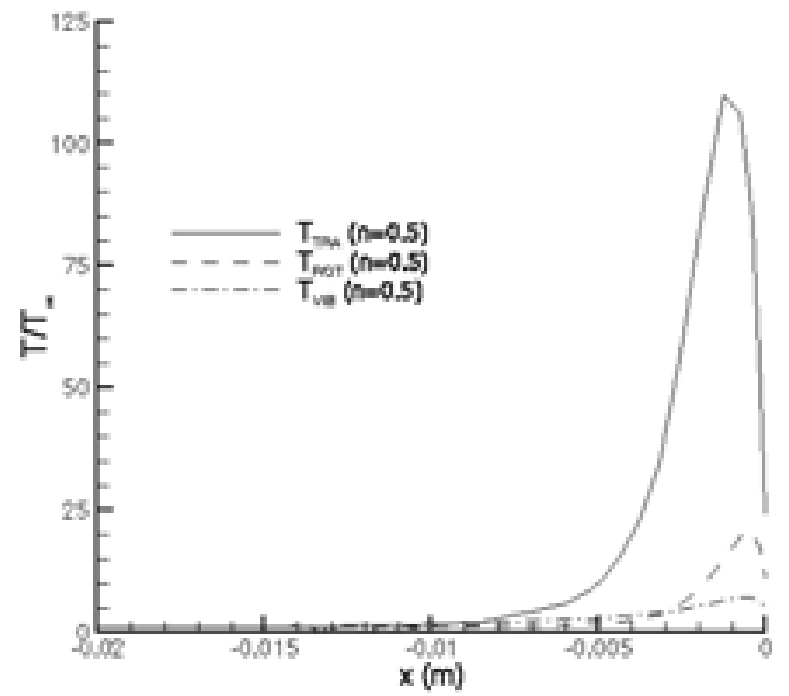

Fig. 7e. Temperature profiles along the stagnation streamline at $\mathrm{U}_{\infty}=7.5 \mathrm{~km} / \mathrm{s}$ for $\mathrm{n}=0.5$.

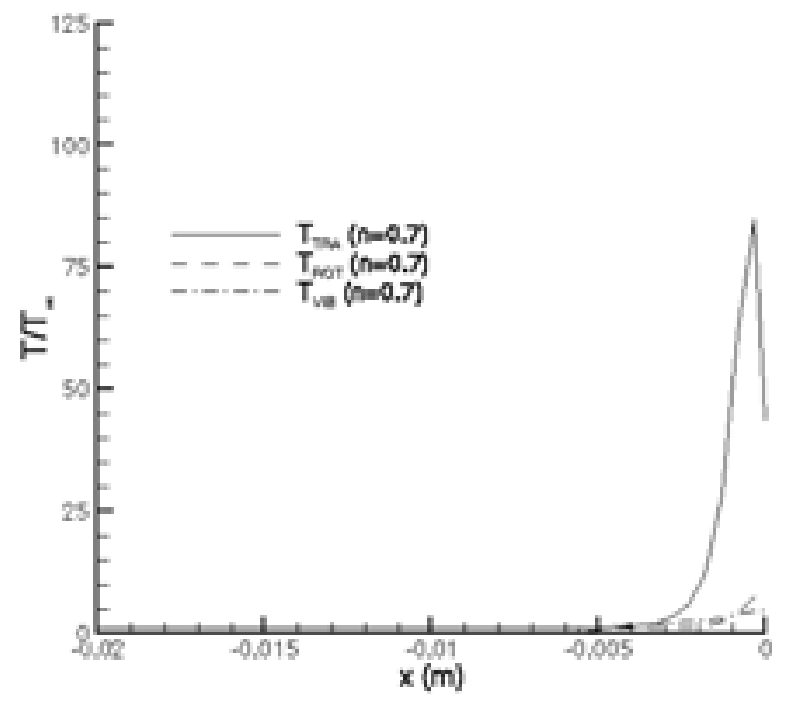

Fig. 7f. Temperature profiles along the stagnation streamline at $\mathrm{U}_{\infty}=7.5 \mathrm{~km} / \mathrm{s}$ for $\mathrm{n}=0.7$.

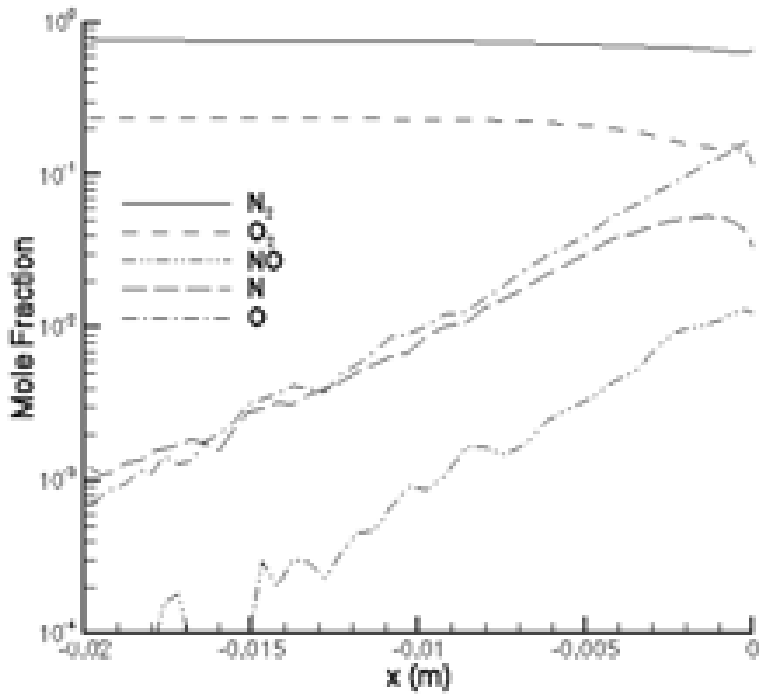

Fig. $7 \mathrm{~g}$. Mole fraction profiles along the stagnation streamline at $\mathrm{U}_{\infty}=7.5 \mathrm{~km} / \mathrm{s}$ for the cylinder.

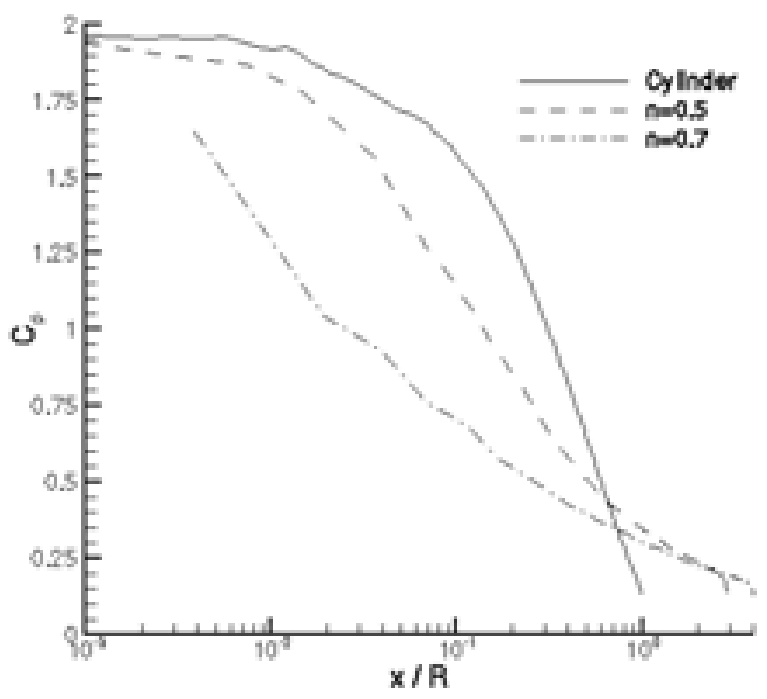

Fig. 8a. Surface profiles of pressure coefficient around various leading edge shapes at $U_{\infty}=7.5 \mathrm{~km} / \mathrm{s}$. 


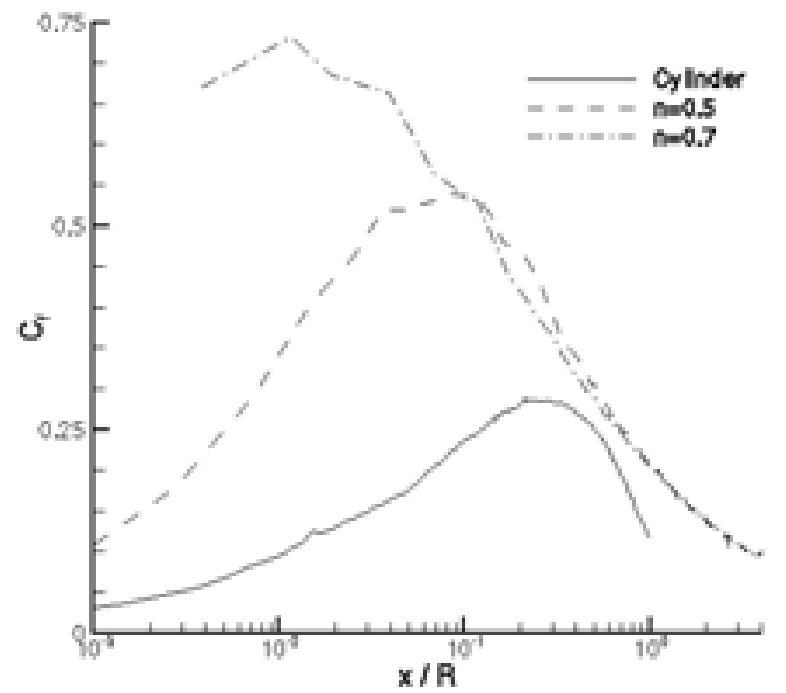

Fig. $8 \mathrm{~b}$. Surface profiles of skin friction coefficient around various leading edge shapes at $\mathrm{U}_{\infty}=7.5 \mathrm{~km} / \mathrm{s}$.

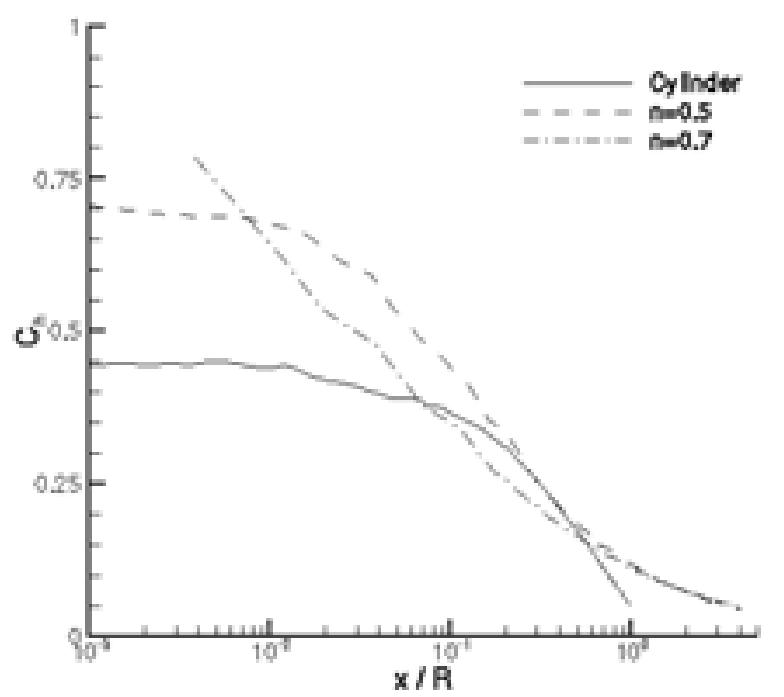

Fig. 8c. Surface profiles of heat transfer coefficient around various leading edge shapes at $U_{\infty}=7.5 \mathrm{~km} / \mathrm{s}$.

The surface properties for the three shapes are compared in Figs. 8a through 8c. Note in all three plots that it is difficult to obtain information at very small $\mathrm{x}$ coordinate for the $n=0.7$ power-law shape due to its very sharp profile. It should be noted that the mesh resolution employed in this case at the tip involves cells that are much smaller than the local mean free path. The profiles of the coefficient of pressure indicate similarities between the cylinder and $n=0.5$ cases, and again each of these is close to the Newtonian model. The $\mathrm{n}=0.7$ case is very different as the pressure does not reach its stagnation value at the tip. The profiles for the skin friction and heat transfer shown in Figs. $8 \mathrm{~b}$ and 8c, respectively, follow the expected trends. The sharper shape of the $\mathrm{n}=0.7$ power-law leads to increased friction and heat transfer at the tip.

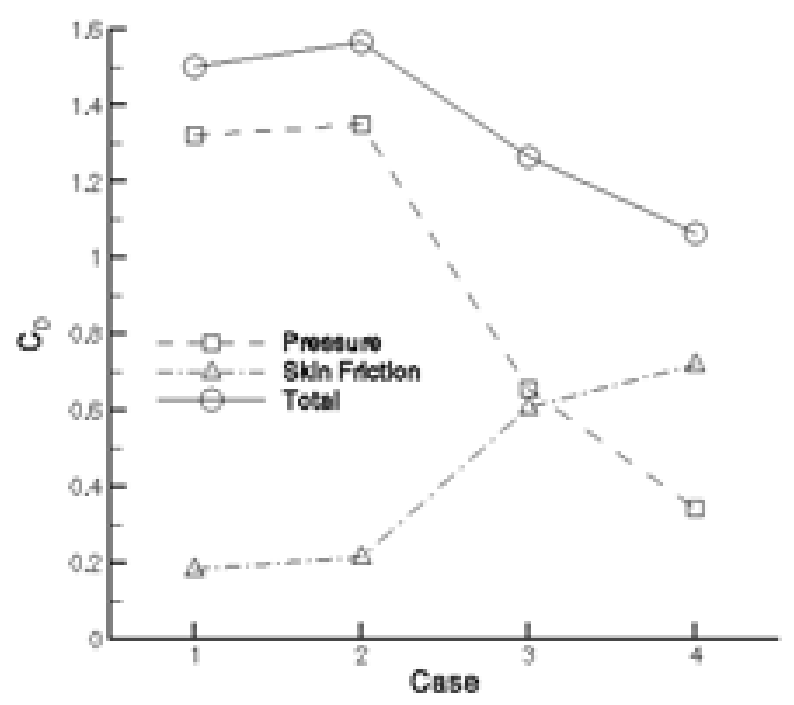

Fig. 9a. Summary of drag data for all cases considered.

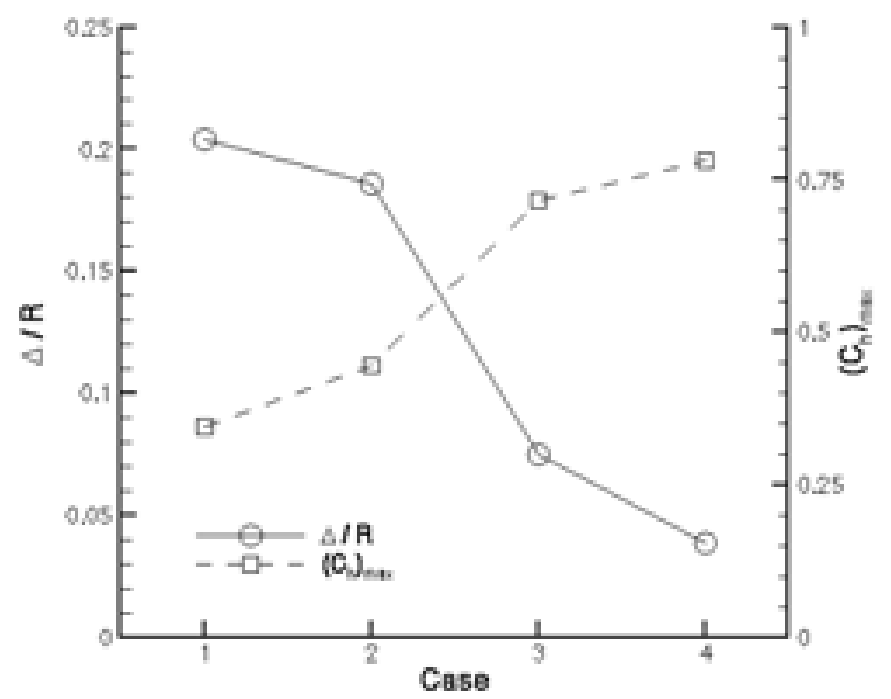

Fig. 9b. Summary of shock standoff and maximum heat transfer coefficient data for all cases considered.

A summary of the computed drag data for all four cases is provided in Fig. 9a. The contributions from pressure and skin friction are shown along with the overall drag. Several conclusions can be drawn from these data. The values obtained for the $3.5 \mathrm{~km} / \mathrm{s}$ cylinder case are almost identical to those reported by Santos and Lewis [3]. For the same cylinder, the drag coefficients are almost identical at the two velocities considered. At the free stream velocity of $7.5 \mathrm{~km} / \mathrm{s}$, making the leading edge sharper leads to: (1) significant decreases in pressure drag (by more than a factor of four); (2) significant increases in skin friction drag (by more than a factor of three); 
(3) modest decreases in overall drag (by about 40\%). A summary plot of the data for shock standoff distance normalized by the body radius, $(\Delta / R)$, and maximum heat transfer coefficient are shown in Fig. 9b. These data clearly illustrate the advantages and disadvantages of sharper leading edge shapes. With the $\mathrm{n}=0.7$ power-law shape, the shock standoff distance is reduced almost to zero, but this is accompanied by a factor of two increase in maximum heat transfer coefficient.

\section{Summary}

The direct simulation Monte Carlo (DSMC) method was used to study the aerothermodynamic performance of sharp leading edges at high altitude, hypersonic flow conditions. These simulations produced a great deal of detailed information about the flow fields and surface properties for the conditions considered. The first stage of the study investigated the effect of free stream velocity for a cylindrical leading edge. It was found that the profiles of normalized density and velocity along the stagnation streamline were unchanged by the free stream velocity. This indicates that the shock standoff distance (when characterized by density ratio) is unaffected by the free stream velocity. The surface profile of pressure coefficient was also

\section{Acknowledgments}

Financial support for this work is provided by the NASAURETI on Reusable Launch Vehicles, Grant NCC-3989. The authors gratefully acknowledge helpful discussions with Professor Mark J. Lewis.

\section{References}

${ }^{1}$ Mason, W.H. and Lee, J., "Aerodynamically Blunt and Sharp Bodies," Journal of Spacecraft and Rockets, Vol. 31, 1994, pp. 378-382.

${ }^{2}$ O'Brien, T.F. and Lewis, M.J., "Power-Law Shapes for Leading-Edge Blunting With Minimal Shock Standoff," Journal of Spacecraft and Rockets, Vol. 36, 1999, pp. 653658.

${ }^{3}$ Santos, W.F.N. and Lewis, M.J., "Power-Law Shaped Leading Edges in Rarefied Hypersonic Flow," Journal of Spacecraft and Rockets, Vol. 39, 2002, pp. 917-925.

${ }^{4}$ Santos, W.F.N. and Lewis, M.J., "Aerodynamic Heating Performance of Power-Law Shaped Leading Edges in Rarefied Hypersonic Flow," AIAA Paper 2003-3894, June 2003.

${ }^{5}$ Bird, G.A.,, Molecular Gas Dynamics and the Direct Simulation of Gas Flows, Oxford Science Publications, New York, 1994.

${ }^{6}$ Bertin, J.J., Hypersonic Aerothermodynamics, AIAA Press, 1994, p. 2.

${ }^{7}$ Detra, R.W., Kemp, N.H., and Riddell, F.R., "Addendum to Heat Transfer to Satellite Vehicles Reentering the Atmosphere," Jet Propulsion, Vol. 27, 1957, pp. 1256-1257.

${ }^{8}$ Dietrich, S. and Boyd, I.D., "Scalar and Parallel Optimized Implementation of the Direct Simulation Monte Carlo Method," Journal of Computational Physics, Vol. 126, 1996, pp. 328-342. unaffected by the free stream velocity. As predicted by the heat transfer correlation of Detra et al. [7], higher heat transfer coefficients were computed at the higher free stream velocity. Qualitatively, the same trend was found for the skin friction coefficient.

The second stage of the study considered the effects of changing the leading edge shape at near-orbital velocity. The expected trends were obtained. Namely, that the sharp powerlaw shape generated much less compression near its tip, resulting in reduced shock standoff distance and pressure coefficient. These improvements in aerothermodynamic performance were, however, offset by increases in the heat transfer and skin friction at the tip of this leading edge.

As with prior leading edge studies $[3,4]$, the present investigation provides quantitative data that can be used to design leading edge shapes. This design process inevitably involves a compromise between reduced drag and shock standoff distance, and increased heat transfer. The present investigation represents just the first step in a lengthy analysis of these issues. Some of the parameters that will be varied in future studies include altitude, leading edge geometry, surface temperature, and angle of attack. We are also interested in investigation of the larger effects of sharp leading edges such as any influence on boundary layer transition and separation.

${ }^{9}$ Wang, W.-L. and Boyd, I.D., "Predicting Continuum Breakdown in Hypersonic Viscous Flows," Physics of Fluids, Vol. 15, 2003, pp. 91-100.

${ }^{10}$ Kannenberg, K.C. and Boyd, I.D., "Three Dimensional Monte Carlo Simulations of Plume Impingement," Journal of Thermophysics and Heat Transfer, Vol. 13, 1999, pp. 226235.

${ }^{11}$ Karipides, D.P., Boyd, I.D. and Caledonia, G.E., "Detailed Simulation of Surface Chemistry Leading to Spacecraft Glow," Journal of Spacecraft and Rockets, Vol. 36, 1999, pp. 566572.

${ }^{12}$ Balakrishnan, J., Boyd, I.D., and Braun, D.G., "Monte Carlo Simulation of Vapor Transport in Physical Vapor Deposition of Titanium," Journal of Vacuum Science and Technology A, Vol. 18, 2000, pp. 907-916.

${ }^{13}$ Boyd, I.D., "Computation of Atmospheric Entry Flow About a Leonid Meteoroid," Earth, Moon, and Planets, Vol. 82, 2000, pp. 93-108.

${ }^{14}$ Koura, K. and Matsumoto, H., "Variable Soft Sphere Molecular Model for Air Species," Physics of Fluids A, Vol. 4, 1992, pp. 1083-1085.

${ }^{15}$ Boyd, I.D., "Analysis of Rotational Nonequilibrium in Standing Shock Waves of Nitrogen," AIAA Journal, Vol. 28, 1990, pp. 1997-1999.

${ }^{16}$ Vijayakumar, P., Sun, Q. and Boyd, I.D., "Detailed Models of Vibrational-Translational Energy Exchange for the Direct Simulation Monte Carlo Method," Physics of Fluids, Vol. 11, 1999, pp. 2117-2126. 